\title{
Role of Drug-Gene Interactions and Pharmacogenetics in Simvastatin-Associated Pulmonary Toxicity
}

\author{
Naomi T. Jessurun ${ }^{1,2} \cdot$ Marjolein Drent ${ }^{2,3,4}$ - Petal A. Wijnen ${ }^{2,4,5} \cdot$ Ankie M. Harmsze ${ }^{4,6}$. \\ Eugène P. van Puijenbroek ${ }^{1,7}$. Otto Bekers ${ }^{2,5}$. Aalt Bast $^{2,3,8}$
}

Accepted: 28 July 2021 / Published online: 4 October 2021

(c) The Author(s) 2021

\begin{abstract}
Introduction Simvastatin has previously been associated with drug-induced interstitial lung disease. In this retrospective observational study, cases with non-specific interstitial pneumonia (NSIP) or idiopathic pulmonary fibrosis (IPF) with simvastatin-associated pulmonary toxicity $(n=34)$ were evaluated.

Objective To identify whether variations in genes encoding cytochrome P450 (CYP) enzymes or in the SLCO1B1 gene (Solute Carrier Organic anion transporting polypeptide 1B1 gene, encoding the organic anion transporting polypeptide 1B1 [OATP1B1] drug transporter enzyme), and/or characteristics of concomitantly used drugs, predispose patients to simvastatinassociated pulmonary toxicity.

Methods Characteristics of concomitantly used drugs and/or variations in the CYP or SLCOIB1 genes and drug-gene interactions were assessed. The outcome after withdrawal of simvastatin and/or switch to another statin was assessed after 6 months. Results Multiple drug use involving either substrates and/or inhibitors of CYP3A4 and/or three or more drugs with the potential to cause acidosis explained the simvastatin-associated toxicity in $70.5 \%(n=24)$ of cases. Cases did not differ significantly from controls regarding CYP3A4, CYP2C9, or OATP1B1 phenotypes, and genetic variation explained only $20.6 \%$ $(n=7)$ of cases. Withdrawal of simvastatin without switching to another statin or with a switch to a hydrophilic statin led to improvement or stabilization in all NSIP cases, whereas all cases who were switched to the lipophilic atorvastatin progressed. Conclusion Simvastatin-associated pulmonary toxicity is multifactorial. For patients with this drug-induced pulmonary toxicity who need to continue taking a statin, switching to a hydrophilic statin should be considered.
\end{abstract}

ClinicalTrials.gov identifier NCT00267800, registered in 2005.

\section{Introduction}

Simvastatin is one of the most successful representatives of the cholesterol-lowering HMG-CoA reductase inhibitors, the class of drugs known as statins [1]. The drug is usually well tolerated, although statin therapy is associated

Marjolein Drent

m.drent@ildcare.nl

1 Netherlands Pharmacovigilance Centre Lareb, 's-Hertogenbosch, The Netherlands

2 ILD Care Foundation Research Team, Ede, The Netherlands

3 Department of Pharmacology and Toxicology, Faculty of Health, Medicine and Life Science, Maastricht University, Maastricht, The Netherlands

4 ILD Center of Excellence, Department of Respiratory Medicine, St. Antonius Hospital, Nieuwegein, The Netherlands with several adverse effects on hepatic, renal, and muscular systems [2]. Adverse reactions in skeletal muscle have been described and range from myalgia (pain) and myopathy (pain with evidence of muscle degradation) to rhabdomyolysis (severe muscle damage which may sometimes cause acute kidney injury) [3]. The widespread use of simvastatin has also revealed several rare and sometimes severe toxicities

5 Department of Clinical Chemistry, Central Diagnostic Laboratory, Maastricht University Medical Centre, Maastricht, The Netherlands

6 Department of Clinical Pharmacy, St. Antonius Hospital, Nieuwegein, The Netherlands

7 Groningen Research Institute of Pharmacy, University of Groningen, Groningen, The Netherlands

8 Venlo Campus, Maastricht University, Venlo, The Netherlands 


\section{Key Points}

This study demonstrates that simvastatin-associated pulmonary toxicity is multifactorial and under-recognized in clinical practice.

It is crucial to consider the metabolic properties of concomitantly used drug(s) in explaining drug adverse effects including simvastatin toxicity, in addition to genetic variations.

in patients. So far, pulmonary toxicity has been reported mainly in case reports or case series [4-7]. The mechanisms behind simvastatin-associated pulmonary toxicity have not yet been elucidated.

A literature review revealed several isolated cases of statin-associated interstitial lung disease (ILD) involving simvastatin-associated pulmonary toxicity. The authors reported idiopathic pulmonary fibrosis (IPF), a severe form of ILD, and non-specific interstitial pneumonia (NSIP) [4-7]. Moreover, $\mathrm{Xu}$ et al. reported that statin use was associated with ILD among smokers in the COPDGene study [8].
The mechanisms and pathophysiology of toxicity associated with statins are still not fully clear, but include both patient-related factors such as age and pharmacogenetics, and factors that impact the pharmacologic, physicochemical, and pleiotropic characteristics of statins [9]. Statins are administered in lactone (e.g. simvastatin, lovastatin) and hydroxy acid forms (e.g. atorvastatin, fluvastatin, pravastatin) and have a complex metabolic pathway (see Fig. 1). After intake, the interconversion of the two forms depends on the $\mathrm{pH}$ in the environment [9-11]. The lactone forms of statins are more cytotoxic to muscular cells than the hydroxy acid forms, and shifts in the ratio between the lactone and hydroxy acid forms may have an effect on pharmacological and toxicological response [9]. After absorption, statins are transported into hepatocytes by organic anion transport polypeptides (OATPs) and metabolized by cytochrome $\mathrm{P} 450$ (CYP) enzymes [12]. Genes encoding drug transporters and drug metabolizing enzymes are subject to polymorphisms, which may affect pharmacokinetics and serum drug levels, and subsequently have an impact on the degree of efficacy and toxicity $[12,13]$.

Genome-wide association studies showed that polymorphisms in the Solute Carrier Organic anion transporting polypeptide 1B1 gene (SLCO1B1, the gene encoding the OATP1B1 transporter) were associated with a higher risk of

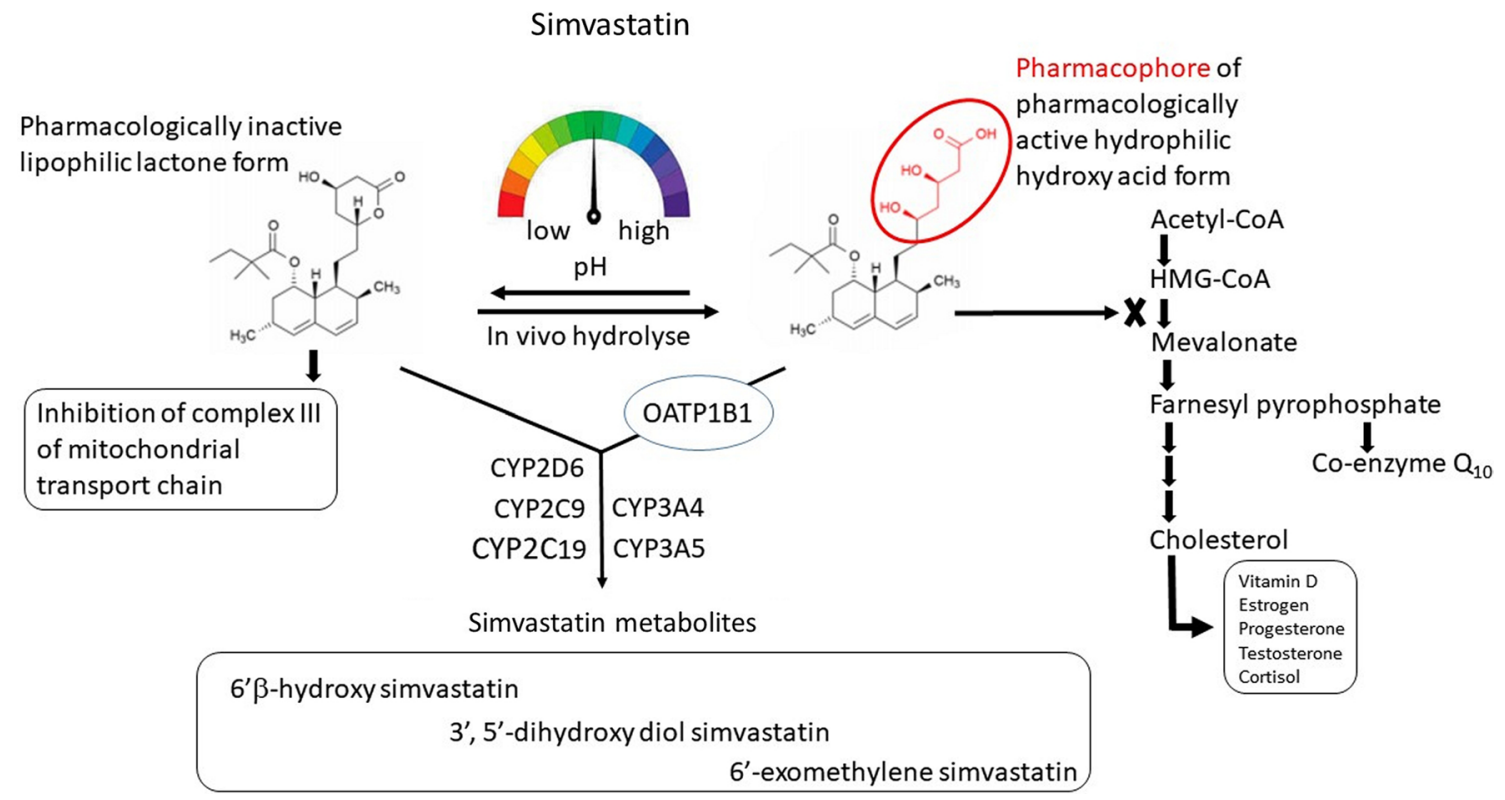

Fig. 1 Simvastatin is administered in the pharmacologically inactive lactone form. In vivo, the lactone hydrolyzes to the corresponding cholesterol-lowering hydroxy acid form. The lactone form is the more lipophilic form and is predominantly present in an acidic environment. An acid-base imbalance may shift the lactone/hydroxy acid ratio [9-11]. Organic anion transport polypeptide 1B1 (OATP1B1) is an uptake transporter expressed on the sinusoidal (basolateral) side of hepatocytes. Simvastatin, like several other statins, is a substrate for this transporter. In hepatocytes, various cytochrome P450 isoenzymes play a role in the biotransformation of simvastatin into various metabolites. It has been suggested that the lactone form inhibits complex III in the mitochondrial electron transport chain. In addition to inhibition of cholesterol formation, statins also hamper the biosynthesis of co-enzyme Q10, which is also critically involved in mitochondrial respiration 
simvastatin toxicity [14]. Moreover, recommended change(s) in the medical management of simvastatin include testing the $S L C O 1 B 1$ genotype [15]. Previously, Li et al. demonstrated that genotype-guided statin therapy may improve patients' perceptions of statins and physician behavior, promoting higher statin adherence [16]. Furthermore, concomitantly used drugs may inhibit and/or compete for the available enzymes, which may also affect the risk/benefit ratio. Inhibitors of drug metabolizing enzymes, such as paroxetine for CYP2D6, are able to transiently convert the phenotype of patients (so-called phenoconversion) and impact the pharmacokinetics of drugs metabolized or transported by the enzymes concerned $[17,18]$. So far, none of these mechanisms and factors in statin toxicity have been extrapolated to explain pulmonary toxicity.

\section{Aim}

The aim of the present retrospective observational study was to assess the possible involvement of concomitantly used drugs, as well as genetic variations in drug metabolizing enzymes and/or drug transporters involved in the metabolism of simvastatin, in patients with simvastatin-associated pulmonary toxicity. In addition, we evaluated the effect of withdrawal of simvastatin and/or its replacement by either a hydrophilic statin (pravastatin, rosuvastatin, fluvastatin) or a lipophilic statin (atorvastatin), on the outcome and course of pulmonary toxicity.

\section{Methods}

\subsection{Study Design and Ethical Statement}

In this retrospective observational study of genotyped patients with NSIP or IPF, we assessed whether characteristics of concomitantly used drugs and/or variations in genes encoding CYP enzymes or in the $S L C O 1 B 1$ gene predispose patients to simvastatin-associated pulmonary toxicity. The study was conducted according to the principles of the Declaration of Helsinki (version 5, 2004) and in accordance with the Dutch Medical Research Involving Human Subjects Act (WMO). The protocol was approved by the Medical research Ethics Committees United (MEC-U) of the St. Antonius Hospital (approval R05-08A). Written informed consent for participation in this study was obtained from all subjects.

ClinicalTrials.gov identifier NCT00267800, registered in 2005 .

\subsection{Selection of Patients and Controls}

Patients who were referred between 2010 and 2019 to the ILD Center of Excellence at St. Antonius Hospital, Nieuwegein, the Netherlands (a tertiary referral center) with established ILD—either NSIP $(n=233)$ or IPF $(n=276)$ and had been genotyped were considered for this observational study (Fig. 2). For patients with unexplained ILD and a history of drug use, genotyping of certain specified genes was included in the diagnostic work-up. A multidisciplinary team confirmed the diagnosis based on clinical presentation, including dyspnea and hypoxia, pulmonary function impairment, exercise intolerance, and high-resolution computed tomography (HRCT) scan abnormalities, including multifocal areas of ground-glass opacity with intralobular interstitial thickening [19].

\subsubsection{Inclusion Criteria}

Patients who used simvastatin $(n=176)$ were considered for this study. Only those who stopped or switched statin treatment were included $(n=34)$. Patients stopped without replacement or switched to a hydrophilic statin or a

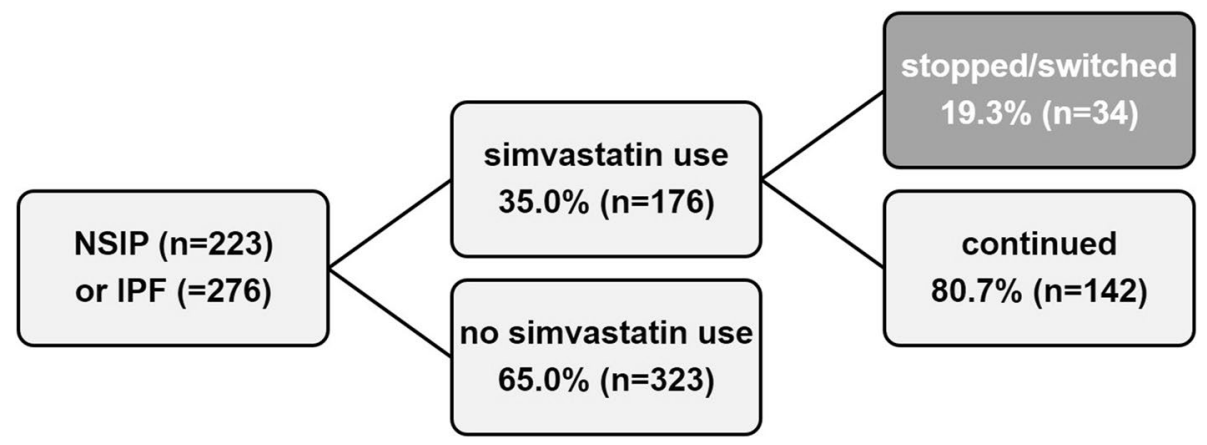

Fig. 2 Flowchart of case selection: cases with non-specific interstitial pneumonitis (NSIP) or idiopathic pulmonary fibrosis (IPF) were divided into those who did not use simvastatin (not included) and those who used simvastatin. Those who used simvastatin were further divided into those who had stopped or switched to another statin (included, $n=34$ ) and those who continued simvastatin (not included, $n=142$ ) 
lipophilic statin. They had already stopped simvastatin use or stopped simvastatin use shortly after referral.

\subsubsection{Exclusion Criteria}

Patients who continued simvastatin, as well as those with other possible causes of pulmonary damage, such as infections or sarcoidosis, were meticulously excluded.

To compare the distribution of the allelic variants between cases (all Caucasian) and the general Caucasian population, controls were collected from the literature [20-25].

\subsection{Data Collection}

Demographic information on the included cases (i.e. gender, age at diagnosis, and concomitant drug use at diagnosis) were collected where necessary. Data on drug use were recorded and retrospectively supplemented with pharmacy data recorded in the electronic health records of the hospital. Concomitantly used drugs were classified according to their metabolic pathways as inhibitors and/or substrates for the enzymes studied. All cases were treated with standard dosages as used in clinical practice. No patient was treated with an unusually high dosage of any of the drugs used. Furthermore, the potential of the concomitantly used drugs to acidify the blood was assessed. The characteristics of the metabolic pathways of the concomitantly used drugs are summarized in Table 1. Appendix A provides a more extensive summary of these characteristics (electronic supplementary material [ESM]).

The outcome or course of the ILD three months after diagnosis was assessed and recorded. Since the cases had been referred to the ILD Center of Excellence, the simvastatin treatment had already been stopped or reduced in most cases. Moreover, as determining serum simvastatin concentration is not standard practice in the Netherlands, no serum drug levels were available or could be obtained.

\subsection{Genotyping}

In all subjects, genomic DNA had been isolated from venous EDTA-anticoagulated blood. CYP2C9 (CYP2C $9 * 2$ [C430T], CYP2C9*3 [A1075C]), CYP2C19 (CYP2C19*2 [G681A], CYP2C19*3 [G636A], CYP2C19*17 [C806T]), CYP2D6 (CYP2D6*3 [A2549del], CYP2D6*4 [G1846A], CYP2D6*6 [T1707del], CYP2D6*5 [del] and CYP2D6 copy number variation), $C Y P 3 A 4$ (CYP3A4*1B [A-392G], CYP3A4*22 [C522-191T]), and SLCO1B1 (SLCO1B1*5 [T521C]) alleles were identified by real-time PCR using the StepOnePlus ${ }^{\mathrm{TM}}$ Real-Time PCR System and TaqMan GTXpress Master/ Drug Metabolizing Genotyping Assay mixes (Applied Biosystems, Foster City, CA, USA), according to the manufacturer's instructions.
In accordance with conventional classification systems, individuals were classified (CYP2D6, CYP2C9, CYP3A4, and CYP2C19) as poor metabolizers (PMs) if they carried two non-functional alleles; as intermediate metabolizers (IMs) if they carried one non-functional allele; as normal metabolizers (NMs) if they carried one allele associated with reduced or increased activity and one functional allele or two functional alleles, and as ultra-rapid metabolizers (UMs) if they carried at least two copies of a functional allele plus a reduced activity allele, two copies of an increased function allele $\left(C Y P 2 C 19^{*} 17\right)$ or three or more copies of a functional allele. SLCO1B1 [521T/T] was classified as having normal transporter capacity, $S L C O 1 B 1$ [521T/C] as reduced transporter capacity, and $S L C O 1 B 1[521 \mathrm{C} / \mathrm{C}]$ as very low transporter capacity. The laboratory that performed the tests is certified (ISO 15189:2012).

\subsection{Data Analysis}

Concomitantly used drugs were evaluated to assess whether they were substrates, inhibitors or inducers of CYP3A4, CYP2D6, CYP2C19, and CYP2C9, and also whether they were OATP1B1 inhibitors or substrates. In addition, their potential to acidify blood was also evaluated. The characteristics of the concomitantly used drugs were categorized in terms of risk factors. Risk factors for simvastatin toxicity were based on previous research and expert opinion and were defined as using two or more drugs that are CYP3A4 substrates; using at least one drug that inhibits CYP3A4 [18]; being a PM or IM of CYP3A4; carrying one or two SLCO1B1 521C alleles [14]; and using three or more drugs that have the potential to cause acidosis [9]. We assessed the concomitantly used drugs, the pharmacogenetic profile, and the number of risk factors for each case.

To compare the distribution of the allelic variants between cases (all Caucasian) and the general Caucasian population, historical controls were collected from the literature [21-23, 25].

To assess the effect of withdrawal of simvastatin after six months, we retrospectively evaluated the medical records of the 34 included cases.

Those patients who had used simvastatin $(n=34)$ and stopped or switched to another statin were considered for this observational study (Fig. 2). Patients were categorized into those who stopped using simvastatin without replacement and those who switched to a hydrophilic statin or to a lipophilic statin. Improvement was considered to have occurred if the forced vital capacity (FVC) had increased by $10 \%$ or more and/or the HRCT had improved. Stabilization was considered to have occurred if the FVC was stable ( $<10 \%$ increase or decrease), while deterioration/progression was considered to have occurred if FVC had decreased 
Table 1 Summary of characteristics and metabolic pathways of concomitantly used drugs [26-29]

\begin{tabular}{|c|c|c|c|c|c|c|c|c|c|}
\hline & $\begin{array}{l}\text { CYP2D6 } \\
\text { INH }\end{array}$ & $\begin{array}{l}\text { CYP2D6 } \\
\text { IND }\end{array}$ & $\begin{array}{l}\text { CYP2D6 } \\
\text { SUB }\end{array}$ & $\begin{array}{l}\text { CYP3A4 } \\
\text { INH }\end{array}$ & $\begin{array}{l}\text { CYP3A4 } \\
\text { IND }\end{array}$ & $\begin{array}{l}\text { CYP3A4 } \\
\text { SUB }\end{array}$ & $\begin{array}{l}\text { OATP1B1 } \\
\text { INH }\end{array}$ & $\begin{array}{l}\text { OATP1B1 } \\
\text { SUB }\end{array}$ & $\begin{array}{l}\text { Acidosis } \\
\text { potential }\end{array}$ \\
\hline \multicolumn{10}{|l|}{ Analgesics } \\
\hline Codeine & 0 & 0 & 1 & 0 & 0 & 0 & 0 & 0 & 0 \\
\hline Diclofenac & 0 & 0 & 0 & 0 & 0 & 0 & 0 & 0 & 1 \\
\hline Morphine & 0 & 0 & 1 & 0 & 0 & 0 & 0 & 0 & 1 \\
\hline Oxycodone; tramadol & 0 & 0 & 1 & 0 & 0 & 1 & 0 & 0 & 0 \\
\hline \multicolumn{10}{|l|}{ Antacids } \\
\hline (es)Omeprazole; pantoprazole & 0 & 0 & 0 & 0 & 0 & 1 & 0 & 0 & 0 \\
\hline \multicolumn{10}{|l|}{ Antibiotics } \\
\hline Sulfamethoxazole/trimethoprim & 0 & 0 & 0 & 0 & 0 & 0 & 0 & 0 & 1 \\
\hline \multicolumn{10}{|l|}{ Antidiabetics } \\
\hline Gliclazide; glimepiride; tolbutamide & 0 & 0 & 0 & 0 & 0 & 0 & 0 & 0 & 1 \\
\hline \multicolumn{10}{|l|}{ Antihypertensives } \\
\hline Bisoprolol; formoterol & 0 & 0 & 1 & 0 & 0 & 0 & 0 & 0 & 1 \\
\hline Enalapril & 0 & 0 & 0 & 0 & 0 & 0 & 0 & 1 & 1 \\
\hline Losartan & 0 & 0 & 0 & 0 & 0 & 1 & 0 & 0 & 0 \\
\hline Metoprolol; timolol & 0 & 0 & 1 & 0 & 0 & 1 & 0 & 0 & 0 \\
\hline Nifedipine & 0 & 0 & 1 & 0 & 0 & 1 & 0 & 0 & 1 \\
\hline Spironolactone & 0 & 0 & 0 & 0 & 0 & 1 & 0 & 0 & 1 \\
\hline Valsartan & 0 & 0 & 0 & 0 & 0 & 0 & 0 & 1 & 0 \\
\hline Verapamil & 0 & 0 & 0 & 1 & 0 & 1 & 0 & 0 & 0 \\
\hline \multicolumn{10}{|l|}{ Antilipemics } \\
\hline Ezetimibe & 0 & 0 & 0 & 0 & 0 & 0 & 0 & 1 & 0 \\
\hline Simvastatin & 0 & 0 & 0 & 0 & 0 & 1 & 0 & 1 & 0 \\
\hline \multicolumn{10}{|l|}{ Antiparkinson agent } \\
\hline Ropinirole & 0 & 0 & 0 & 0 & 0 & 1 & 0 & 0 & 0 \\
\hline \multicolumn{10}{|l|}{ Antithrombotics } \\
\hline Acenocoumarol; phenprocoumon & 0 & 0 & 0 & 0 & 0 & 1 & 0 & 0 & 0 \\
\hline \multicolumn{10}{|l|}{ Corticosteroids } \\
\hline Prednisolone; prednisone & 0 & 0 & 0 & 0 & 0 & 1 & 0 & 0 & 0 \\
\hline \multicolumn{10}{|l|}{ Psychotropics } \\
\hline Amitriptyline & 0 & 0 & 1 & 0 & 0 & 0 & 0 & 0 & 1 \\
\hline Escitalopram & 0 & 0 & 1 & 0 & 0 & 1 & 0 & 0 & 0 \\
\hline Haloperidol & 0 & 0 & 1 & 0 & 0 & 1 & 0 & 0 & 1 \\
\hline Temazepam; zolpidem & 0 & 0 & 0 & 0 & 0 & 1 & 0 & 0 & 0 \\
\hline \multicolumn{10}{|l|}{ Others } \\
\hline Flecainide & 0 & 0 & 1 & 0 & 0 & 0 & 0 & 0 & 0 \\
\hline Tacrolimus & 0 & 0 & 0 & 0 & 0 & 1 & 0 & 0 & 1 \\
\hline Tamsulosin & 0 & 0 & 1 & 0 & 0 & 1 & 0 & 0 & 0 \\
\hline
\end{tabular}

IND induction, INH inhibition, $S U B$ substrate

by $>10 \%$ and the HRCT demonstrated progression of the features $[30,31]$.

\subsection{Statistical Analysis}

Differences in CYP3A4, CYP2D6, CYP2C9, CYP2C19, and $S L C O 1 B 1$ genotype frequencies between cases and controls were assessed using a Fisher exact test in R (version 3.5.1, Vienna, Austria) [32]. Actual allele distributions were compared with the expected frequencies that were calculated using the Hardy-Weinberg (HW) equilibrium. Deviations from HW were analyzed using the chi-squared test. A Bonferroni correction was applied to adjust for multiple 
comparisons; a $p$ value $<0.01$ was considered to be statistically significant.

\section{Results}

In a sample of NSIP and IPF cases ( $n=499$; average age [ \pm SD]: 69.0 [ \pm 9.5] years; 342 [68.5\%] men; 157 [31.5\%] women), a total of 176 cases (136 [77.3\%] men; 40 [22.7\%]) women) were identified as using simvastatin. Of these 176 cases, 34 (19.3\% [34/176]; including seven women [20.6\%]), with an average age $( \pm$ SD) of $69.2( \pm 8.2)$ years, were found to have stopped simvastatin use due to suspected pulmonary toxicity (i.e., deterioration occurred after simvastatin was started). There was no age difference between the original sample of NSIP and IPF cases $(n=499)$ and the 34 identified cases that had stopped simvastatin. Although overall fewer women used simvastatin, the percentage of women who developed pulmonary toxicity did not differ from that of the male patients. The percentage of cases who had stopped simvastatin due to simvastatin-associated pulmonary toxicity did not differ between men (19.6\%) and women (17.5\%). The period of drug use varied from six months to 10 years, with a median of five years. None of the cases had used simvastatin in a dosage above $40 \mathrm{mg}$ daily. Based on the available diagnostic tests and exclusion of other possible diagnostic options, the diagnosis was highly likely.

Diagnoses and outcomes of the 34 cases are summarized in Table 2.

\subsection{Drug-Gene Interactions and Pharmacogenetics}

Information on the concomitantly used drugs and phenotypes of the cases is summarized in Tables 2, 3, and 4. As regards drug interactions and pharmacogenetics, the majority of cases $(82.4 \%)$ had several possible risk factors
Table 2 Demographics, genetic data and concomitantly used drugs of the studied population with non-specific interstitial pneumonitis (NSIP) or idiopathic pulmonary fibrosis (IPF)

\begin{tabular}{|c|c|c|c|}
\hline & NSIP & IPF & Total \\
\hline Number of cases (\%) & $16(47.1 \%)$ & $18(52.9 \%)$ & $34(100 \%)$ \\
\hline Age, years $( \pm S D)$ & $68.6( \pm 9.5)$ & $69.7( \pm 7.1)$ & $69.2( \pm 8.2)$ \\
\hline Gender, female (\%) & $5(31.2 \%)$ & $2(11.1 \%)$ & $7(20.6 \%)$ \\
\hline Simvastatin stopped, no switch to other statin (\%) & 7 & 5 & $12(35.3 \%)$ \\
\hline Improvement & 6 & 0 & 6 \\
\hline Stable & 1 & 5 & 6 \\
\hline Progression & 0 & 0 & 0 \\
\hline Simvastatin stopped, switch to hydrophilic statin (\%) & 6 & 6 & $12(35.3 \%)$ \\
\hline Improvement & 3 & 0 & 3 \\
\hline Stable & 3 & 5 & 8 \\
\hline Progression & 0 & $\begin{array}{r}1 \text { (stable, later } \\
\text { progression) }\end{array}$ & 1 \\
\hline Simvastatin stopped, switch to lipophilic statin (\%) & 3 & 7 & $10(29.4 \%)$ \\
\hline Improvement & 0 & 0 & 0 \\
\hline Stable & 0 & 0 & 0 \\
\hline Progression & 3 & 7 & 10 \\
\hline $\begin{array}{l}S L C O 1 B 1 \text { polymorphisms (reduced transporter capacity or } \\
\text { very low transporter capacity) (\%) [not analyzed] }\end{array}$ & $3[3]$ & $3[5]$ & $6(23.1 \%)$ \\
\hline Concomitant use of OATP1B1 inhibitors/substrates & 0 & 0 & 0 \\
\hline CYP3A4 polymorphisms (IM) (\%) & 1 & 1 & $2(5.9 \%)$ \\
\hline Concomitant use of CYP3A4 inhibitors/substrates & 1 & 1 & 2 \\
\hline$C Y P 2 D 6$ polymorphisms (IM or PM) (\%) & 7 & 10 & $17(50.0 \%)$ \\
\hline Concomitant use of CYP2D6 inhibitors/substrates & 5 & 5 & 10 \\
\hline$C Y P 2 C 9$ polymorphisms (IM or PM) $(\%)$ & 8 & 8 & $16(47.1 \%)$ \\
\hline Concomitant use of CYP2C9 inhibitors/substrates & 6 & 5 & 13 \\
\hline CYP2C19 polymorphisms (IM or PM) (\%) & 6 & 5 & $11(32.4 \%)$ \\
\hline Concomitant use of CYP2C19 inhibitors/substrates & 4 & 2 & 6 \\
\hline Concomitant use of medication that induces acidosis (\%) & 17 & 15 & $32(94.1 \%)$ \\
\hline Patients using $\geq 3$ drugs that induce acidosis & 4 & 9 & 13 \\
\hline
\end{tabular}

CYP cytochrome P450, IM intermediate metabolizer, OATP1B1 organic anion transporting polypeptide 1B1, $P M$ poor metabolizer, $S D$ standard deviation, $S L C O 1 B 1$ solute carrier organic anion transporting polypeptide $1 \mathrm{~B} 1$ 
Table 3 Individual cases, pharmacogenetics, possible mechanisms of interacting drugs and risk factors that may affect simvastatin-associated toxicity (marked in red)

\begin{tabular}{|c|c|c|c|c|c|c|c|c|c|c|c|c|c|c|c|c|c|c|c|c|}
\hline Nr. & Gender & Diagnosis & \begin{tabular}{|l|} 
Age \\
(years)
\end{tabular} & Course & \begin{tabular}{|l|} 
2D6 \\
INH \\
\end{tabular} & \begin{tabular}{|l|} 
2D6 \\
SUB \\
\end{tabular} & 2D6 & \begin{tabular}{|l|} 
3A4 \\
INH
\end{tabular} & \begin{tabular}{|l|} 
3A4 \\
SUB
\end{tabular} & $3 \mathrm{A4}$ & \begin{tabular}{|l|} 
2C9 \\
INH \\
\end{tabular} & \begin{tabular}{|l|} 
2C9 \\
SUB \\
\end{tabular} & $2 \mathrm{C9}$ & \begin{tabular}{|l|} 
2C19 \\
INH
\end{tabular} & \begin{tabular}{|l|}
2 C19 \\
SUB
\end{tabular} & $2 \mathrm{C} 19$ & \begin{tabular}{|l|} 
OATP1B1 \\
INH
\end{tabular} & \begin{tabular}{|l|} 
OATP1B1 \\
SUB
\end{tabular} & \begin{tabular}{|l|} 
SLCO1B1 \\
T521C
\end{tabular} & \begin{tabular}{|l|} 
Drugs with \\
potential to \\
acidify blood
\end{tabular} \\
\hline \multicolumn{21}{|c|}{ Simvastatin stopped, no switch to another statin } \\
\hline 1. & M & NSIP & 79 & improved & 0 & 1 & NM & 1 & 5 & NM & 0 & 1 & IM & 1 & 1 & NM & 0 & 1 & $\mathrm{~T} / \mathrm{T}$ & 3 \\
\hline 2. & F & NSIP & 71 & improved & 0 & 0 & PM & 0 & 4 & NM & 0 & 2 & IM & 0 & 0 & IM & 0 & 2 & n/a & 2 \\
\hline 3. & M & NSIP & 68 & improved & 0 & 3 & IM & 0 & 3 & NM & 0 & 2 & IM & 1 & 1 & NM & 0 & 1 & $\mathrm{~T} / \mathrm{C}$ & 2 \\
\hline 4. & F & NSIP & 76 & improved & 0 & 0 & IM & 0 & 2 & NM & 0 & 0 & IM & 0 & 0 & NM & 0 & 1 & n/a & 1 \\
\hline 5. & M & NSIP & 69 & improved & 0 & 2 & NM & 0 & 3 & NM & 0 & 1 & NM & 0 & 0 & NM & 0 & 1 & $\mathrm{~T} / \mathrm{T}$ & 3 \\
\hline 6. & M & NSIP & 74 & improved & 0 & 0 & NM & 0 & 2 & NM & 0 & 1 & NM & 1 & 2 & NM & 0 & 2 & $T / T$ & 0 \\
\hline 7. & M & NSIP & 86 & stable & 0 & 2 & IM & 0 & 5 & NM & 0 & 1 & IM & 1 & 2 & NM & 0 & 2 & $T / T$ & 2 \\
\hline 8. & M & IPF & 70 & stable & 0 & 1 & IM & 0 & 2 & NM & 0 & 1 & NM & 0 & 0 & IM & 0 & 1 & $T / T$ & 2 \\
\hline 9. & M & IPF & 75 & stable & 0 & 2 & IM & 0 & 3 & NM & 0 & 0 & IM & 0 & 0 & NM & 0 & 1 & n/a & 3 \\
\hline 10. & M & IPF & 58 & \begin{tabular}{|l|l|} 
stable \\
\end{tabular} & 0 & 0 & IM & 0 & 1 & NM & 0 & 1 & NM & 0 & 0 & PM & 0 & 1 & T/C & 3 \\
\hline 11. & $\mathrm{~F}$ & IPF & 82 & stable & 0 & 0 & IM & 0 & 1 & NM & 0 & 1 & NM & 0 & 0 & NM & 0 & 2 & n/a & 2 \\
\hline 12. & M & IPF & 69 & stable & 0 & 1 & IM & 0 & 4 & NM & 0 & 0 & NM & 1 & 1 & NM & 0 & 1 & $\mathrm{~T} / \mathrm{T}$ & 1 \\
\hline \multicolumn{21}{|c|}{ Simvastatin stopped, switched to a hydrophilic statin } \\
\hline 13. & M & NSIP & 63 & \begin{tabular}{|l|}
$\begin{array}{l}\text { rosuvastatin } \\
\text { improved }\end{array}$ \\
\end{tabular} & 0 & 2 & IM & 0 & & NM & 0 & 2 & NM & 0 & 0 & IM & 0 & 1 & $\mathrm{~T} / \mathrm{T}$ & 2 \\
\hline 14. & M & NSIP & 68 & \begin{tabular}{|l|} 
pravastatin \\
improved
\end{tabular} & 0 & 1 & NM & 0 & 2 & NM & 0 & 0 & NM & 0 & 0 & NM & 0 & 2 & $T / T$ & 1 \\
\hline 15. & F & NSIP & 67 & \begin{tabular}{|l|}
$\begin{array}{l}\text { fluvastatin } \\
\text { improved }\end{array}$ \\
\end{tabular} & 0 & 0 & NM & 0 & 2 & NM & 1 & 2 & IM & 1 & 1 & IM & 0 & 1 & T/C & 2 \\
\hline 16. & M & NSIP & 68 & \begin{tabular}{|l|}
$\begin{array}{l}\text { rosuvastatin } \\
\text { stable }\end{array}$ \\
\end{tabular} & 0 & 1 & NM & 0 & & NM & 0 & 2 & NM & 1 & 1 & NM & 0 & 1 & $\mathrm{~T} / \mathrm{T}$ & 1 \\
\hline 17. & F & NSIP & 61 & \begin{tabular}{|l} 
rosuvastatin \\
stable
\end{tabular} & 0 & 1 & NM & & 2 & NM & 0 & 0 & IM & 0 & 0 & NM & 0 & 1 & n/a & 3 \\
\hline 18. & M & NSIP & 71 & \begin{tabular}{|l|}
$\begin{array}{l}\text { pravastatin } \\
\text { stable }\end{array}$ \\
\end{tabular} & 0 & 2 & NM & & 7 & IM & 1 & 2 & NM & 1 & 2 & IM & 0 & 2 & T/T & 2 \\
\hline 19. & M & IPF & 72 & $\begin{array}{l}\begin{array}{l}\text { rosuvastatin } \\
\text { stable }\end{array} \\
\end{array}$ & 0 & 1 & IM & 0 & & NM & 0 & 1 & NM & 1 & 1 & NM & 0 & 1 & $\mathrm{~T} / \mathrm{T}$ & 2 \\
\hline 20. & M & IPF & 75 & $\begin{array}{l}\text { rosuvastatin } \\
\text { stable }\end{array}$ & 0 & 0 & IM & 0 & 1 & NM & 0 & 1 & PM & 0 & 0 & NM & 0 & 1 & $\mathrm{~T} / \mathrm{T}$ & \\
\hline 21. & M & IPF & 72 & \begin{tabular}{|l|} 
rosuvastatin \\
stable
\end{tabular} & 0 & 0 & IM & 0 & 1 & NM & 0 & 0 & NM & 0 & 0 & NM & 0 & 1 & $\mathrm{~T} / \mathrm{T}$ & 4 \\
\hline 22. & M & IPF & 65 & \begin{tabular}{|l|}
$\begin{array}{l}\text { rosuvastatin } \\
\text { stable }\end{array}$ \\
\end{tabular} & 0 & 1 & IM & 0 & 2 & IM & 0 & 0 & NM & 0 & 0 & NM & 0 & 1 & $\mathrm{~T} / \mathrm{T}$ & 2 \\
\hline 23. & M & IPF & 74 & \begin{tabular}{|l|}
$\begin{array}{l}\text { rosuvastatin } \\
\text { stable }\end{array}$ \\
\end{tabular} & 0 & 0 & IM & 0 & 1 & NM & 0 & 1 & IM & 0 & 1 & NM & 0 & 1 & n/a & 3 \\
\hline 24. & M & IPF & 72 & \begin{tabular}{|l|} 
rosuvastatin \\
stable, later \\
progression
\end{tabular} & 0 & 1 & NM & 0 & & NM & 1 & 1 & IM & 1 & 2 & IM & 0 & 1 & $T / T$ & \\
\hline \multicolumn{21}{|c|}{ Simvastatin stopped, switched to atorvastatin } \\
\hline 25. & M & NSIP & 58 & progression & 0 & 1 & NM & 0 & 2 & NM & 2 & 1 & NM & 0 & 1 & IM & 0 & 2 & $\mathrm{~T} / \mathrm{T}$ & 3 \\
\hline 26. & $\mathrm{~F}$ & NSIP & 44 & progression & 0 & 1 & IM & & 4 & NM & 0 & 1 & IM & 1 & 1 & IM & 0 & 2 & $\mathrm{~T} / \mathrm{T}$ & 1 \\
\hline 27. & M & NSIP & 74 & progression & 0 & 1 & IM & 0 & 4 & NM & 0 & 2 & NM & 1 & 3 & NM & 0 & 1 & $\mathrm{~T} / \mathrm{C}$ & 2 \\
\hline 28. & M & IPF & 78 & progression & 0 & 1 & NM & 0 & & NM & 0 & 2 & IM & 0 & 1 & NM & 0 & 1 & n/a & 1 \\
\hline 29. & F & IPF & 69 & progression & 0 & 1 & NM & 0 & 2 & NM & 0 & 0 & NM & 0 & 0 & NM & 0 & 1 & $\mathrm{~T} / \mathrm{T}$ & 2 \\
\hline 30. & M & IPF & 58 & progression & 0 & 0 & NM & 0 & 2 & NM & 1 & 1 & NM & 0 & 1 & UM & 0 & 1 & $n / a$ & 0 \\
\hline 31. & M & IPF & 63 & progression & 0 & 1 & NM & 0 & & NM & 0 & 0 & IM & 0 & 0 & IM & 0 & 1 & $T / T$ & 3 \\
\hline 32. & M & IPF & 72 & progression & 0 & 1 & IM & 0 & & NM & 0 & 1 & NM & 1 & 1 & NM & 0 & 1 & T/C & 4 \\
\hline 33. & M & IPF & 56 & progression & 0 & 1 & IM & 0 & 2 & NM & 0 & 1 & IM & 0 & 1 & IM & 0 & 1 & $T / T$ & 3 \\
\hline 34. & M & IPF & 75 & progression & 0 & 2 & NM & 0 & 2 & NM & 0 & 0 & IM & 0 & 1 & IM & 0 & 1 & $T / C$ & 2 \\
\hline
\end{tabular}

$F$ female, IPF idiopathic pulmonary fibrosis, IM intermediate metabolizer, $I N H$ number of concomitantly used drugs that inhibit the CYP enzyme, $M$ male, $n / a$ not applicable, $N M$ normal metabolizer, $N S I P$ non-specific interstitial pneumonitis, $P M$ poor metabolizer, $U M$ ultra-rapid metabolizer, OATP1B1 organic anion transporting polypeptide 1B1, SLCO1B1 solute carrier organic anion transporter 1B1, SLCO1B1 521T/T wildtype; SLCO1B1 521T/C heterozygous variant, SUB number of concomitantly used drugs that are metabolized by this enzyme 
Table 4 Metabolic genotype frequencies of CYP3A4, CYP2C9, CYP2D6, CYP2C19, and SLOC1B1 in cases and controls [15-18]

\begin{tabular}{|c|c|c|}
\hline$C Y P 3 A 4(p=0.45)$ & Cases $(n=34)$ & $\begin{array}{l}\text { Historical controls } \\
(n=235)[21]\end{array}$ \\
\hline Poor metabolizer & $0(0.0 \%)$ & $0(0.0 \%)$ \\
\hline Intermediate metabolizer & $2(5.9 \%)$ & $20(8.5 \%)$ \\
\hline Normal metabolizer & $32(94.1 \%)$ & $215(91.5 \%)$ \\
\hline$C Y P 2 C 9(p=0.20)$ & Cases $(n=34)$ & Controls $(n=121)[22]$ \\
\hline Poor metabolizer & $1(2.9 \%)$ & $3(2.5 \%)$ \\
\hline Intermediate metabolizer & $15(44.2 \%)$ & $36(29.8 \%)$ \\
\hline Normal metabolizer & $18(52.9 \%)$ & $82(67.8 \%)$ \\
\hline$C Y P 2 D 6(p=0.03)$ & Cases $(n=34)$ & Controls $(n=765)$ [23] \\
\hline Poor metabolizer & $1(2.9 \%)$ & $42(5.5 \%)$ \\
\hline Intermediate metabolizer & $18(52.9 \%)$ & $233(30.3 \%)$ \\
\hline Normal metabolizer & $15(44.2 \%)$ & $490(64.2 \%)$ \\
\hline Ultra-rapid metabolizer & $0(0.0 \%)$ & $0(0.0 \%)$ \\
\hline$C Y P 2 C 19(p=0.04)$ & Cases $(n=34)$ & Controls $(n=736)$ [23] \\
\hline Poor metabolizer & $1(2.9 \%)$ & $19(2.6 \%)$ \\
\hline Intermediate metabolizer & $10(29.4 \%)$ & $163(22.1 \%)$ \\
\hline Normal metabolizer & $22(64.7 \%)$ & $554(75.3 \%)$ \\
\hline Ultra-rapid metabolizer & $1(2.9 \%)$ & $0(0.0 \%)$ \\
\hline $\operatorname{SLCO1B1}(p=0.88)$ & Cases $(n=26)$ & Controls $(n=724)[25]$ \\
\hline Very low transporter capacity & $0(0.0 \%)$ & $12(1.5 \%)$ \\
\hline Reduced transporter capacity & $6(23.1 \%)$ & $193(26.5)$ \\
\hline
\end{tabular}

CYP cytochrome P450, SLCO1B1 solute carrier organic anion transporting polypeptide 1B1

assumed to be associated with simvastatin toxicity, and only six cases (17.6\%: cases $4,8,11,14,29$, and 30) had none of these risk factors (Table 3 ). These cases all concomitantly used two drugs inhibiting CYP3A4, which might have played a role in the development of simvastatin toxicity. The OATP1B1 activity of three cases was unknown. Multiple use of drugs that are substrates and/or inhibitors of CYP3A4, and/or three or more drugs that have the potential to cause acidosis, explained the simvastatin-associated toxicity in $70.5 \%$ of cases. Genetic variation causing a reduced OATP1B1 activity and/or being an IM of CYP3A4 explained toxicity in $20.6 \%$. In two cases, the only risk factor was reduced OATP1B1 activity, whereas in one case it was being an IM of CYP3A4. The risk factors assessed are summarized in Table 5.

All four cases who used a drug inhibiting CYP3A4 concomitantly used two or more other drugs, and up to seven drugs that are CYP3A4 substrates. Six cases had reduced OATP1B1 activity, but none of them used a substrate or inhibitor of the drug transporter, apart from simvastatin. Two of these cases used three or more CYP3A4 substrates (see Table 3).

The actual allele distributions of the cases were in HW equilibrium. However, the cases tended to differ from the
Table 5 Extent to which cases $(n=34)$ were exposed to possible risk factors that may affect simvastatin-associated toxicity

\begin{tabular}{lc}
\hline Risk factors & $\begin{array}{l}\text { Number of } \\
\text { cases, } n(\%)\end{array}$ \\
\hline Using two or more other drugs that are CYP3A4 substrates & $17(50.0)$ \\
Using at least one drug that inhibits CYP3A4 & $4(11.8)$ \\
Being an intermediate or poor CYP3A4 metabolizer & $2(5.9)$ \\
SLCO1B1 521T/C heterozygote or SLCO1B1 521C/C homozygous variant genotype & $6(23.1)$ \\
$(n=26,8$ not analyzed) & $13(38.2)$ \\
\hline
\end{tabular}

CYP cytochrome P450, SLCO1B1 solute carrier organic anion transporting polypeptide 1B1, SLCO1B1 $521 T / C$ heterozygote, SLCO1B1 521C/C homozygous variant 
controls, with more PMs and/or IMs for CYP2D6 $(p=0.03)$ and $C Y P 2 C 19(p=0.04)$. Cases did not differ significantly from controls in phenotypes of CYP3A4 $(p=0.45), C Y P 2 C 9$ $(p=0.20)$, or SLCO1B1 $(p=0.88)$. More than half of the cases with reduced metabolic activity concomitantly used drugs that compete with and/or inhibit the metabolic enzymes concerned (see Table 4).

\subsection{Outcomes After Withdrawal of Simvastatin}

Twelve cases had stopped simvastatin use without continuing with another statin, while 12 cases had switched to a hydrophilic statin (rosuvastatin nine; pravastatin two; fluvastatin one) and 10 had switched to the lipophilic statin atorvastatin. Cases with NSIP who had stopped simvastatin without replacing it with another statin were more likely to improve (85.7\%) than those who had simvastatin replaced by a hydrophilic statin (50.0\%). The five IPF cases who stopped all stabilized, which in the case of IPF is the best that is achievable. No improvement was observed in cases who were switched to atorvastatin.

\section{Discussion}

In this retrospective study, we identified 34 cases with simvastatin-associated pulmonary toxicity. In our population, at least 34 out of 176 simvastatin users (19.3\%) developed simvastatin-induced ILD. It is important, however, to realize that the number of simvastatin users in the general population is much larger, and our sample is influenced by selection bias. It is therefore hard to give a reliable indication of simvastatin-associated toxicity in the whole population of simvastatin users. However, the cases we studied showed a remarkable association. The fact that most of the NSIP cases (6/7) improved or stabilized (1/7) after withdrawal of the drug makes this association highly likely.

Drug interactions are a substantial cause of adverse effects, leading to hospitalization and sometimes to death [33]. Estimating the interaction potential of concomitantly used drugs is difficult. Drugs can be substrates, inhibitors or inducers of several drug metabolizing enzymes and drug transporters, and pathways may play major or minor roles in the biotransformation of the drug involved [34]. We assessed whether concomitantly used drugs could interact with the biotransformation of simvastatin and hence predispose patients to simvastatin-associated pulmonary toxicity. In the population studied, concomitant use of multiple drugs is quite common. Polypharmacy may lead to various drug-drug interactions. Moreover, we observed that almost all cases (90\%) used at least one drug that was a substrate for CYP3A4, and that $35.3 \%$ used two or more drugs, while $50 \%$ used three or more. This is also considered the most important metabolic pathway for simvastatin. More than two-thirds of the cases (70\%) used one or more drugs that may cause acidosis, which in turn may enhance the formation of the more toxic lactone form of simvastatin.

Simvastatin is available and administered in the lactone prodrug form. Depending on the local $\mathrm{pH}$, simvastatin is primarily present in either the pharmacologically active hydroxy acid form or the pharmacologically inactive and more lipophilic lactone form [9]. Disturbances in the acid/base balance of the blood impact the interconversion between the more lipophilic lactone and the more hydrophilic hydroxy acid form of simvastatin, and so have an effect on drug concentrations and the observed toxicity of simvastatin $[9,35]$. Various metabolites of simvastatin also contain the lactone moiety and will undergo a similar $\mathrm{pH}-$ dependent interconversion (Fig. 1). Furthermore, the lactone form disturbs complex III in the mitochondrial transport chain. In an acidic environment (i.e. at a low $\mathrm{pH}$ ), more of the lactone form is present, which is also associated with a higher incidence and severity of statin-induced myotoxicity [10].

There are various conditions that may lead to acidosis. Metabolic acidosis can occur when too much of the basic compounds is lost. This can be caused by diarrhea, kidney damage or the use of cholesterol-lowering agents. Extreme exercise can lead to lactate acidosis, and insufficiently controlled type 1 diabetes mellitus may also result in acidosis. Furthermore, acidosis may be caused by intoxication with alcohol, while dysfunctional lung physiology may lead to so-called respiratory acidosis. It is also known that the $\mathrm{pH}$ drops at inflammation sites. This means that more of the lactone form will be present at an existing inflammation site (e.g. in the lung).

The lipophilicity of simvastatin increases as the $\mathrm{pH}$ drops, as is indicated by the increase in $\log \mathrm{P}$ octanol/water, which

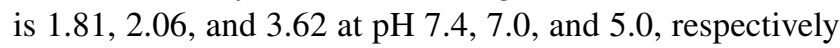
[35]. Moreover, drug-induced acidification may also shift the lactone versus hydroxy acid equilibrium in favor of the toxic lactone form [10]. Drugs that cause metabolic acidosis can be grouped into four categories: drugs that represent exogenous acid loads (e.g. salicylates); drugs leading to loss of bicarbonate in the gastrointestinal tract or kidney (e.g. topiramate); drugs causing increased endogenous acid production (e.g. metformin or isoniazid, which may lead to lactic acid, or paracetamol and beta-lactam penicillin, leading to pyroglutamic acid); drugs that compromise renal acid secretion (e.g. drugs that act via inhibition of the reninangiotensin-aldosterone system, or via impaired proximal or distal tubule $\mathrm{H}+$-secretion, as is the case with acetazolamide or lithium) [36]. Most of our cases (94.1\%) concomitantly used drugs that have the potential to at least slightly lower the blood $\mathrm{pH}$. Although previous findings point to a relationship between the incidence and severity of statin-induced 
myopathy and a more acidic blood, or more lipophilic statins, it is too early to extrapolate this to statin-associated pulmonary toxicity [9].

A major factor explaining statin-associated myotoxicity is the dosage, and consequently the concentration, of the drug [37-40]. Polypharmacy may influence the simvastatin concentration because of the possibility of drug-drug interaction. This may also apply to the pulmonary toxicity observed in our cases and is illustrated by the finding that after withdrawal of simvastatin without switching to another statin, the lung function of eight NSIP patients improved, while one of the two IPF patients also improved and the other remained stable. In the cases where the lipophilic simvastatin was replaced by a hydrophilic statin, the lung function either improved or remained stable. By contrast, replacing simvastatin by the lipophilic atorvastatin further worsened the clinical condition in all cases. This corresponds with what has been found for statin-associated myotoxicity, which is related to the use of the more lipophilic statins simvastatin and atorvastatin [41].

Genetic variation is also a risk factor, though to a lesser extent. Both SLCO1B1 and CYP3A4 appeared to be important in explaining simvastatin-associated DI-ILD. There are known risk factors for the more extensively investigated statin-associated myotoxicity, which include advanced age, female gender, drug interactions, genetic variability in drug metabolizing enzymes and transporters, lipophilicity of statins, coincident morbidities, and high doses of the statin used [42]. By contrast, risk factors for pulmonary toxicity are largely unknown.

We observed that variations in genes encoding enzymes known to play a role in simvastatin metabolism (CYP3A4 and/or OATP1B1) did not differ between cases and controls. Variations in genes encoding CYP enzymes and transport proteins influence the pharmacokinetics and toxicity of simvastatin, which is mainly biotransformed by CYP3A4 and transported into the hepatocytes by OATP1B 1 [43]. Using human liver microsomes, it has been shown by means of both immune inhibition and classical inhibitor studies that CYP3A4/5 is primarily involved ( $>80 \%$ ) in the metabolism of simvastatin hydroxy acid. A minor contribution to the simvastatin metabolism $(<20 \%)$ was found for CYP2C8 [44]. The allelic frequencies of the two most important $C Y P 3 A 4$ variants $(C Y P 3 A 4 * 22$ and $C Y P 3 A 4 * 1 B)$ are low and have a limited role in the interindividual differences in CYP3A4 expression and activity [45]. Other sources, such as epigenetic factors, should also be considered. Furthermore, external factors such as medication (CYP3A4 inhibitors) and nutrition (grapefruit juice) may reduce the metabolic activity of CYP3A4, resulting in transient poor metabolism due to phenoconversion [18]. This is why we checked for CYP3A4 polymorphism and co-administered drugs, which act either as competitive substrates or as inhibitors of CYP3A4. Although only two of our cases had CYP3A4 polymorphisms, and the phenotypes did not differ significantly from healthy controls, most of our cases used several CYP3A4 substrates and inhibitors. One of the two cases who were genotyped as IMs used six other CYP3A4 substrates and two CYP3A4 inhibitors (patient 18). Such a situation may lead to increased simvastatin concentrations. Moreover, it is not only the number of drugs taken that is relevant, but also their dosages. A high dose of a single inhibitor may result in the same toxicity as a normal to low dose of multiple drugs metabolized by the same affected enzyme.

The exact role of CYP2D6 in the biotransformation of simvastatin is not yet clear, but some studies point to a relationship between being a PM and having more Type A plasma-level dependent adverse drug reactions (ADRs), or between being a UM and having less cholesterol lowering effects [46-48]. Interestingly, 19 out of our 34 cases (55.9\%) had CYP2D6 polymorphisms (PM or IM), which is more than might be expected from data for the general population, and tended to differ from the controls [49]. However, this did not identify risk factors related to the use of drugs that have the potential to be a CYP2D6 substrate.

The protein OATP1B1 is not only important in transporting endogenous compounds like bile acids, steroids, and hormones, but also for the transport of drugs like statins, ACE inhibitors, or methotrexate [29]. Polymorphisms in $S L C O 1 B 1$, its encoding gene, result in altered transport of statins and their metabolites into the liver. Several studies have demonstrated that SLCO1B1 T521C significantly affects simvastatin pharmacokinetics, causing decreased transport into hepatocytes, increased serum simvastatin levels and increased risk of myopathy [50]. Although several of our genotyped cases were carriers of SLCO1B1 521C $(8 / 26)$, they did not differ statistically significantly from the controls in this respect.

In addition to the assessed role of concomitantly used drugs and pharmacogenetics, the mechanism behind statin-associated ILD should be further explored. One of the mechanisms that is associated with lung injury is the formation of reactive oxygen species (ROS) during biotransformation of xenobiotics including pharmaceuticals. This results in the formation of reactive electrophilic species such as epoxides and quinones, which react with cell molecules and cause direct cell toxicity [51-53]. Xu et al. showed that pravastatin and also atorvastatin treatment increases the formation of ROS, which results in increased inflammasomemediated immune response [8]. Furthermore, the generation of ROS during the metabolism of statins and the increase of oxidative stress are associated with well-known ADRs of statins, such as myopathy, nephrotoxicity, hepatotoxicity, and various diabetic complications [54]. The period during which simvastatin had been used varied from six months to 10 years. This underlines that simvastatin is not often 
recognized as a drug associated with pulmonary toxicity. Awareness of the pulmonary toxicity in addition to the better known toxicities like myopathies may avoid more progressive pulmonary damage and benefit patients' quality of life.

One large cohort study has so far been unable to confirm that statins are a significant risk factor in the development of ILD [7]. However, Xu et al. found that statin use is associated with interstitial lung abnormalities among current and former smokers in the COPDGene study [8]. Statin use was positively associated with ILD (odds ratio 1.60 ; 95\% confidence interval $1.03-2.50 ; p=0.04$ ) after adjustment for covariates including a history of high cholesterol or coronary artery disease. Although we were unable to establish a causal relationship between the observed ILD and simvastatin use, we did observe that all cases who had stopped using simvastatin and had not switched to another statin improved, which is in line with other studies with well-documented cases [4-6]. In line with Xu et al. [8], we acknowledge that although increased risks of ILD and radiologic features of pulmonary fibrosis are causes for concern, these risks likely do not outweigh the substantial benefits of statin therapy in patients with cardiovascular disease. Instead, we believe that clinicians should be aware that radiographic evidence of ILD, much like myopathy [3], can occur in some patients on statins.

To verify whether there have been other reports of simvastatin-associated pulmonary toxicity besides what has been published [4-7], we searched EudraVigilance (the system for suspected ADRs in the European Economic Area) for cases of pulmonary toxicity associated with simvastatin. This search yielded more than 200 reports of pulmonary toxicity associated with simvastatin (164 ILD, four IPF, and 59 pulmonary fibrosis cases). Analyzing the data in these reports could contribute to knowledge about the association between simvastatin and ILD, and possibly provide further insight into the risk factors assessed.

Limitations of our study are the rather small number of cases and the fact we did not determine simvastatin serum levels. We deliberately selected only those who stopped simvastatin use, as in these cases the association between the observed toxicity and simvastatin use was acknowledged and was strongest. Because these cases were sent to a referral center, and in most instances simvastatin had already been stopped or stopped shortly after referral, we could not assess serum drug levels. In fact, in the case of simvastatin (hydroxy acid form), which has a very short plasma half-life ( \pm 1.9 hours), assessing the serum drug level makes little sense and is also not standard practice in the Netherlands. Another limitation is that we only used a historical control group of Caucasians to compare polymorphism distribution. A more valid control group would be a sample of cases using simvastatin who did not develop pulmonary abnormalities. It would then be interesting to know which drugs were used concomitantly, especially drugs that may cause acidosis and/ or those that affect the metabolic pathway of simvastatin, and to investigate whether the polymorphism distribution differed from that in our sample. Unfortunately, we do not have access to such a sample. Moreover, cases without pulmonary symptoms will never be referred to our department.

\section{Conclusion}

We demonstrated that simvastatin may cause pulmonary toxicity. Simvastatin-associated pulmonary toxicity is complex, multifactorial, and under-recognized in clinical practice. Therefore, it is highly likely that the number of reported cases is underestimated. Multiple drug use, concomitantly used drugs that inhibit CYP3A4 or are metabolized by CYP3A4, and/or using three or more drugs that may acidify the blood, converting simvastatin to the more lipophilic and more toxic lactone form, explained the simvastatin-associated pulmonary toxicity in $70.5 \%$ of our patients.

Genetic variation, mostly resulting in a reduced OATP1B1 activity or in being an intermediate CYP3A4 metabolizer, explained toxicity in more than $20 \%$ of the cases. It is therefore essential to consider the metabolic properties of concomitantly used drug(s) in explaining toxicity, in addition to genetic variations. It should be realized that polypharmacy by itself may have a huge influence and that the drug interactions may be mistaken for genetic variation. Studies on concomitantly used drugs comparing cases with and without simvastatin-associated ADRs could be a topic for further research. Although we could not establish a firm relationship between the use of simvastatin and the pulmonary toxicity observed in our cases, withdrawal of simvastatin without switching to another statin led to improvement in almost all NSIP cases. This not only points to a potential relationship, but also shows the best clinical strategy. If the use of a statin in patients with simvastatin-associated toxicity is essential, it appears that switching to a hydrophilic statin such as rosuvastatin yields better outcomes than switching to the lipophilic atorvastatin, which yielded the poorest outcomes. The advice that continuation with a hydrophilic statin is a better choice in case of simvastatin-associated toxicity applies not only to myotoxicities, but according to our cases, also to pulmonary toxicities.

Supplementary Information The online version contains supplementary material available at https://doi.org/10.1007/s40264-021-01105-8.

Acknowledgements The authors wish to thank the ILD care foundation (http://www.ildcare.nl) for supporting this research.

\section{Declarations}

Funding This research received no external funding. 
Conflict of interest The authors declare no conflict of interest.

Ethics approval The study was conducted according to the principles of the Declaration of Helsinki (version 5, 2004) and in accordance with the Dutch Medical Research Involving Human Subjects Act (WMO). The protocol was approved by the Medical research Ethics Committees United (MEC-U) of the St. Antonius Hospital (approval R05-08A). The study protocol was approved by the Ethics Committee.

Consent to participate All participants provided written informed consent.

\section{Consent for publication Not applicable.}

Availability of data and material Anonymized data can be made available to investigators upon request to the corresponding author.

\section{Code availability Not applicable.}

Author contributions All authors read and approved the final version. Conceptualization: NTJ, MD, and AB; data curation: MD, PAW, and AMH; methodology: NTJ, MD, PAW, and AMH: writing - original draft: NTJ, MD and AB; writing-review and editing: NTJ, MD, PAW, $\mathrm{AMH}, \mathrm{EPP}, \mathrm{OB}$, and $\mathrm{AB}$.

Open Access This article is licensed under a Creative Commons Attribution-NonCommercial 4.0 International License, which permits any non-commercial use, sharing, adaptation, distribution and reproduction in any medium or format, as long as you give appropriate credit to the original author(s) and the source, provide a link to the Creative Commons licence, and indicate if changes were made. The images or other third party material in this article are included in the article's Creative Commons licence, unless indicated otherwise in a credit line to the material. If material is not included in the article's Creative Commons licence and your intended use is not permitted by statutory regulation or exceeds the permitted use, you will need to obtain permission directly from the copyright holder. To view a copy of this licence, visit http://creativecommons.org/licenses/by-nc/4.0/.

\section{References}

1. Salami JA, Warraich H, Valero-Elizondo J, Spatz ES, Desai NR, Rana JS, et al. National trends in statin use and expenditures in the US adult population from 2002 to 2013: insights from the medical expenditure panel survey. JAMA Cardiol. 2017;1(2):56-65.

2. Collins R, Reith C, Emberson J, Armitage J, Baigent C, Blackwell $\mathrm{L}$, et al. Interpretation of the evidence for the efficacy and safety of statin therapy. Lancet. 2016 Nov;19(388):2532-61.

3. Buettner C, Rippberger MJ, Smith JK, Leveille SG, Davis RB, Mittleman MA. Statin use and musculoskeletal pain among adults with and without arthritis. Am J Med. 2012;125:176-82.

4. Liebhaber MI, Wright RS, Gelberg HJ, Dyer Z, Kupperman JL. Polymyalgia, hypersensitivity pneumonitis and other reactions in patients receiving HMG-CoA reductase inhibitors: a report of ten cases. Chest. 1999;115:886-9.

5. Lantuejoul S, Brambilla E, Brambilla C, Devouassoux G. Statininduced fibrotic nonspecific interstitial pneumonia. Eur Respir J. 2002 Mar;19:577-80.

6. Walker T, McCaffery J, Steinfort C. Potential link between HMG$\mathrm{CoA}$ reductase inhibitor (statin) use and interstitial lung disease. Med J Aust. 2007 Jan;15(186):91-4.

7. Saad N, Camus P, Suissa S, Ernst P. Statins and the risk of interstitial lung disease: a cohort study. Thorax. 2013 Apr;68:361-4.
8. Xu JF, Washko GR, Nakahira K, Hatabu H, Patel AS, Fernandez IE, et al. Statins and pulmonary fibrosis: the potential role of NLRP3 inflammasome activation. Am J Respir Crit Care Med. 2012;1(185):547-56.

9. Taha DA, De Moor CH, Barrett DA, Lee JB, Gandhi RD, Hoo $\mathrm{CW}$, et al. The role of acid-base imbalance in statin-induced myotoxicity. Transl Res. 2016;174:140-60.e14.

10. Taha DA, Zgair A, Lee JB, de Moor CH, Barrett DA, Bruce KD, et al. Hyperlipidaemia alone and in combination with acidosis can increase the incidence and severity of statin-induced myotoxicity. Eur J Pharm Sci. 2017 Mar;30(100):163-75.

11. Fujino H, Saito T, Tsunenari Y, Kojima J, Sakaeda T. Metabolic properties of the acid and lactone forms of HMG-CoA reductase inhibitors. Xenobiotica. 2004;34:961-71.

12. Maggo SD, Kennedy MA, Clark DW. Clinical implications of pharmacogenetic variation on the effects of statins. Drug Saf. 2011;1(34):1-19.

13. Thomas RE. Optimising seniors' metabolism of medications and avoiding adverse drug events using data on how metabolism by their P450 enzymes varies with ancestry and drug-drug and drugdrug-gene interactions. J Pers Med. 2020;11:10.

14. Pasanen MK, Neuvonen M, Neuvonen PJ, Niemi M. SLCO1B1 polymorphism markedly affects the pharmacokinetics of simvastatin acid. Pharmacogenet Genom. 2006;16:873-9.

15. Hippman $C$, Nislow C. Pharmacogenomic testing: clinical evidence and implementation challenges. J Pers Med. 2019;7:9.

16. Li JH, Joy SV, Haga SB, Orlando LA, Kraus WE, Ginsburg GS, et al. Genetically guided statin therapy on statin perceptions, adherence, and cholesterol lowering: a pilot implementation study in primary care patients. J Pers Med. 2014;27(4):147-62.

17. Jessurun NT, Vermeulen Windsant A, Mikes O, van Puijenbroek EP, van Marum RJ, Grootens K, et al. Inhibition of CYP2D6 with low dose $(5 \mathrm{mg})$ paroxetine in patients with high 10-hydroxynortriptyline serum levels-A prospective pharmacokinetic study. $\mathrm{Br}$ J Clin Pharmacol. 2021;87:1529-32.

18. Shah RR, Smith RL. Addressing phenoconversion: the Achilles' heel of personalized medicine. Br J Clin Pharmacol. 2015;79:222-40.

19. Wijsenbeek M, Cottin V. Spectrum of fibrotic lung diseases. N Engl J Med. 2020;3(383):958-68.

20. Wijnen PA, Drent M, Nelemans PJ, Kuijpers PM, Koek GH, Neef $\mathrm{C}$, et al. Role of cytochrome $\mathrm{P} 450$ polymorphisms in the development of pulmonary drug toxicity: a case-control study in the Netherlands. Drug Saf. 2008;2008(31):1125-34.

21. Wang D, Guo Y, Wrighton SA, Cooke GE, Sadee W. Intronic polymorphism in CYP3A4 affects hepatic expression and response to statin drugs. Pharmacogenomics J. 2011 Aug;11:274-86.

22. Allabi AC, Gala JL, Desager JP, Heusterspreute M, Horsmans Y. Genetic polymorphisms of CYP2C9 and CYP2C19 in the Beninese and Belgian populations. Br J Clin Pharmacol. 2003;56:653-7.

23. Tamminga WJ, Wemer J, Oosterhuis B, de Zeeuw RA, de Leij LF, Jonkman JH. The prevalence of CYP2D6 and CYP2C19 genotypes in a population of healthy Dutch volunteers. Eur J Clin Pharmacol. 2001;57:717-22.

24. Sachse C, Brockmöller J, Bauer S, Roots I. Cytochrome P450 2D6 variants in a Caucasian population: allele frequencies and phenotypic consequences. Am J Hum Genet. 1997;60:284-95.

25. Couvert P, Giral P, Dejager S, Gu J, Huby T, Chapman MJ, et al. Association between a frequent allele of the gene encoding OATP1B1 and enhanced LDL-lowering response to fluvastatin therapy. Pharmacogenomics. 2008;9:1217-27.

26. Wijnen PA. Polymorphisms in interstitial lung diseases: friend or foe? Ph.D. Thesis, March 2011; ISBN: 978-90-78076-07-0.

27. Medicines Information Bank. Medicines Evaluation Board. http:// www.cbg-meb.nl. Accessed 29 June 2020. 
28. KNMP Kennisbank. http://www.knmp.nl. Accessed 24 Sept 2020.

29. Kalliokoski A, Niemi M. Impact of OATP transporters on pharmacokinetics. Br J Pharmacol. 2009;158:693-705.

30. Wuyts WA, Wijsenbeek M, Bondue B, Bouros D, Bresser P, Robalo Cordeiro $\mathrm{C}$, et al. Idiopathic pulmonary fibrosis: best practice in monitoring and managing a relentless fibrotic disease. Respiration. 2020;99:73-82.

31. Travis WD, Costabel U, Hansell DM, King TE Jr, Lynch DA, Nicholson AG, et al. An official American Thoracic Society/European Respiratory Society statement: update of the international multidisciplinary classification of the idiopathic interstitial pneumonias. Am J Respir Crit Care Med. 2013;188:733-48.

32. R Core Team. R: a Language and environment for statistical computing; R Foundation for Statistical Computing: Vienna, Austria, 2018.

33. Lazarou J, Pomeranz BH, Corey PN. Incidence of adverse drug reactions in hospitalized patients: a meta-analysis of prospective studies. JAMA. 1998;15(279):1200-5.

34. Jaquenoud Sirot E, van der Velden JW, Rentsch K, Eap CB, Baumann P. Therapeutic drug monitoring and pharmacogenetic tests as tools in pharmacovigilance. Drug Saf. 2006;29:735-68.

35. Joshi HN, Fakes MG, Serajuddin ATM. Differentiation of 3-hydroxy-3-methylglutaryl-coenzyme A reductase inhibitors by their relative lipophilicity. Pharm Pharmacol Commun. 1999;5:269-71.

36. Kitterer D, Schwab M, Alscher MD, Braun N, Latus J. Drug-induced acid-base disorders. Pediatr Nephrol. 2015 Sep;30:1407-23.

37. Hoffman KB, Kraus C, Dimbil M, Golomb BA. A survey of the FDA's AERS database regarding muscle and tendon adverse events linked to the statin drug class. PLoS ONE. 2012;7:e42866.

38. Silva M, Matthews ML, Jarvis C, Nolan NM, Belliveau P, Malloy $\mathrm{M}$, et al. Meta-analysis of drug-induced adverse events associated with intensive-dose statin therapy. Clin Ther. 2007;29:253-60.

39. Cham S, Evans MA, Denenberg JO, Golomb BA. Statin-associated muscle-related adverse effects: a case series of 354 patients. Pharmacotherapy. 2010 Jun;30:541-53.

40. Rallidis LS, Fountoulaki K, Anastasiou-Nana M. Managing the underestimated risk of statin-associated myopathy. Int J Cardiol. 2012 Sep;6(159):169-76.

41. Bruckert E, Hayem G, Dejager S, Yau C, Bégaud B. Mild to moderate muscular symptoms with high-dosage statin therapy in hyperlipidemic patients-the PRIMO study. Cardiovasc Drugs Ther. 2005 Dec;19:403-14.
42. Taha DA, De Moor CH, Barrett DA, Gershkovich P. Translational insight into statin-induced muscle toxicity: from cell culture to clinical studies. Transl Res. 2014 Aug;164:85-109.

43. Williams D, Feely J. Pharmacokinetic-pharmacodynamic drug interactions with HMG-CoA reductase inhibitors. Clin Pharmacokinet. 2002;41:343-70.

44. Prueksaritanont T, Ma B, Yu N. The human hepatic metabolism of simvastatin hydroxy acid is mediated primarily by CYP3A, and not CYP2D6. Br J Clin Pharmacol. 2003;56:120-4.

45. Hirota T, Takane H, Higuchi S, Ieiri I. Epigenetic regulation of genes encoding drug-metabolizing enzymes and transporters; DNA methylation and other mechanisms. Curr Drug Metab. 2008;9:34-8.

46. Mulder AB, van Lijf HJ, Bon MA, van den Bergh FA, Touw DJ, Neef $C$, et al. Association of polymorphism in the cytochrome CYP2D6 and the efficacy and tolerability of simvastatin. Clin Pharmacol Ther. 2001;70:546-51.

47. Nordin C, Dahl ML, Eriksson M, Sjöberg S. Is the cholesterollowering effect of simvastatin influenced by CYP2D6 polymorphism? Lancet. 1997 Jul;5(350):29-30.

48. Zuccaro P, Mombelli G, Calabresi L, Baldassarre D, Palmi I, Sirtori CR. Tolerability of statins is not linked to CYP450 polymorphisms, but reduced CYP2D6 metabolism improves cholesteraemic response to simvastatin and fluvastatin. Pharmacol Res. 2007;55:310-7.

49. Serpe L, Canaparo R, Scordo MG, Spina E. Pharmacogenetics of drug-metabolizing enzymes in Italian populations. Drug Metab Pers Ther. 2015;30:107-20.

50. Kitzmiller JP, Mikulik EB, Dauki AM, Murkherjee C, Luzum JA. Pharmacogenomics of statins: understanding susceptibility to adverse effects. Pharmgenom Pers Med. 2016;9:97-106.

51. Jessurun NT, Drent M, van Puijenbroek EP, Bekers O, Wijnen PA, Bast A. Drug-induced interstitial lung disease: role of pharmacogenetics in predicting cytotoxic mechanisms and risks of side effects. Curr Opin Pulm Med. 2019;25:468-77.

52. Ryrfeldt A. Drug-induced inflammatory responses to the lung. Toxicol Lett. 2000;112-113:171-6.

53. Castell JV, Donato MT, Gomez-Lechon MJ. Metabolism and bioactivation of toxicants in the lung. The in vitro cellular approach. Exp Toxicol Pathol. 2005;57(Suppl 1):189-204.

54. Liu A, Wu Q, Guo J, Ares I, Rodríguez JL, Martínez-Larrañaga MR, et al. Statins: adverse reactions, oxidative stress and metabolic interactions. Pharmacol Ther. 2019;195:54-84. 